\title{
Expression of Yes-associated protein 1 and its clinical significance in ovarian serous cystadenocarcinoma
}

\author{
SANG YEON CHO ${ }^{1 *}$, KWANGHUN KIM ${ }^{2 *}$, MIN SOO PARK ${ }^{1}$, MI YOUNG JANG ${ }^{1}$, YOUNG HWAN CHOI ${ }^{1}$, \\ SUYEON HAN ${ }^{1}$, HYUN MO SHIN $^{2}$, CHAEUK CHUNG $^{3}$, HYE YOUNG HAN $^{4}$, JUNG BO YANG $^{5,6}$, \\ YOUNG BOK KO ${ }^{5,6}$ and HEON JONG YOO ${ }^{5,6}$ \\ ${ }^{1}$ Chungnam National University School of Medicine, Daejeon; ${ }^{2}$ Department of Anatomy, Seoul National University College \\ of Medicine, Seoul; ${ }^{3}$ Department of Pulmonary and Critical Care Medicine, Chungnam National University Hospital; \\ ${ }^{4}$ Department of Pediatrics, Chungnam National University; ${ }^{5}$ Department of Obstetrics and Gynecology, \\ Chungnam National University College of Medicine; ${ }^{6}$ Department of Obstetrics and Gynecology, \\ Chungnam National University Hospital, Daejeon, Republic of Korea
}

Received September 27, 2016; Accepted November 3, 2016

DOI: $10.3892 / o r .2017 .5517$

\begin{abstract}
Yes-associated protein 1 (YAP1) is a key transcriptional regulator in the Hippo signaling pathway that plays a critical role in the development and progression of several types of malignancies, including ovarian cancer. Herein, we investigated the expression of YAP1 and its clinical significance in a large population of patients with ovarian serous cystadenocarcinoma (OSC), which is the most common form of epithelial ovarian neoplasm, using the TCGA database. Surprisingly, cross-cancer mRNA expression and alterations in YAP1 were higher in OSC than in those of other types of cancers in the TCGA database. YAP1 mRNA expression was significantly higher in OSC compared with normal ovarian samples, and was higher in stages III and IV, than stages I and II. The level of YAP1 protein, which is mainly localized to the nucleus, was also higher in stage IV as compared with stages I, II and III. However, the protein level of pYAP1, which is inactive and is localized to the cytoplasm, was not significantly different between stages. The ratio of pYAP/YAP, which shows higher activity at a low ratio, was lower in stage III than in stages I and II. High YAP and low pYAP levels were significantly correlated with a poor prognosis in patients with OSC.
\end{abstract}

Correspondence to: Professor Heon Jong Yoo or Professor Young Bok Ko, Department of Obstetrics and Gynecology, Chungnam National University College of Medicine, Daejeon, Republic of Korea E-mail: bell4184@cnuh.ac.kr

E-mail: koyoung27@cnuh.ac.kr

*Contributed equally

Key words: Yes-associated protein, ovarian serous cystadenocarcinoma, The Cancer Genome Atlas, Hippo signaling pathway, overall survival
The mRNA and protein expression of YAP1 were significantly increased in the proliferative subtype as compared to the differentiated, immunoreactive and mesenchymal subtypes. According to bioinformatics analysis, YAP1 is most highly correlated with the cell cycle. TGF- $\beta$ signaling and WNT signaling were significantly increased in the high YAP1 group according to gene set enrichment analysis. Taken together, our results suggest that not only high YAP1 expression but also its subcellular distribution may be associated with poor overall survival in patients with OSC.

\section{Introduction}

Yes-associated protein (YAP), along with the transcriptional co-activator TAZ, is a main downstream effector of the Hippo pathway, which regulates tissue homeostasis, organ size, regeneration and tumorigenesis (1). In mammalian systems, the Hippo pathway is composed of the core kinase complexes mammalian Ste2-like kinases 1/2 and large tumor suppressor kinases 1/2 (2). The main function of the Hippo pathway is to negatively regulate the activity of YAP and TAZ, to promote cellular proliferation, and to induce anti-apoptotic genes via interactions with various transcription factors (2-4). When the Hippo pathway is active, the inhibitory mammalian Ste2-like kinases/large tumor suppressor kinases phosphorylate YAP and TAZ. Phosphorylation leads to nuclear exclusion of YAP and TAZ. Then, YAP and TAZ are sequestered and subjected to proteasomal degradation in the cytoplasm; also, gene expression of YAP- and TAZ-driven molecules is suppressed $(4,5)$. Overexpression of YAP1 has been found in various types of cancers (6-9), and may lead to oncogenic transformation of immortalized epithelial cells (10). The expression and role of YAP1 in cancer is cell type-dependent $(11,12)$. Overexpression of YAP was observed in $62 \%$ of hepatocellular carcinomas and $72.6 \%$ of colorectal cancers, and was found to be an independent predictor associated with poor disease-free survival and overall survival (13). In $66.3 \%$ of non-small cell lung cancers, YAP was found to be overexpressed, and was 
associated with reduced overall survival (14). Several studies reported that YAP1 is overexpressed in ovarian cancer (6) and acts as an oncogene (15). Zhang et al reported that high levels of nuclear YAP1 correlate with poor prognosis in ovarian cancer patients with clear cell carcinoma (15). Another study showed that YAP1 is highly expressed in serous/endometrioid cystadenocarcinomas, and is positively associated with patient prognosis (16). However, the role of YAP1 as an oncogene has not yet been fully investigated in a large group of ovarian serous cystadenocarcinoma (OSC) patients, who account for the largest proportion of malignant ovarian cancer cases $(17,18)$. Therefore, in the present study, we investigated the expression of YAP1 and determined its clinical significance in OSC.

\section{Materials and methods}

Gene expression profiles. Level 3 mRNA expression data from 8 normal and 590 OSC samples were obtained from the TCGA data portal (https://tcga-data.nci.nih.gov/tcga/).

Analysis of mRNA microarray data. The raw data was initially analyzed using R software (v.3.2.5; http://www.r-project.org/). The chip data was normalized using the RankNormalize module in GenePattern (http://www.broadinstitute.org/cancer/ software/genepattern). GeneNeighbors and ClassNeighbors, modules programmed in GenePattern (http://www.broadinstitute.org/cancer/software/genepattern), were used to select genes closely related to YAP1 (19). cBioportal (http://www. cbioportal.org/) was also used to analyze cross-cancer alterations in YAP1.

Functional enrichment analysis. The DEGs were imported into the Database for Annotation, Visualization and Integrated Discovery (http://david.abcc.ncifcrf.gov/) (20) in order to perform Gene Ontology (GO) functional enrichment analysis. Gene set enrichment analysis (GSEA) was used to enrich the mRNAs predicted to have a correlation with pathway in $\mathrm{C} 2$, curated gene set enrichment analysis $(21,22)$. GO analysis encompasses 3 domains: biological processes, cellular components and molecular functions. $\mathrm{P}<0.05$ was considered to indicate statistical significance.

Statistical analysis. The distributions of characteristics between the 2 groups were compared using the t-test for continuous variables (or the Kolmogorov-Smirnov test when the expected frequency within any cell was $<5$ ), and the $\chi^{2}$ test (or Fisher's exact test when the expected frequency within any cell was $<5$ ) for categorical variables. The distributions of characteristics between 3 or more groups were compared using ANOVA. Cumulative event (death) rate was calculated by the Kaplan-Meier method, using the time to the first event as the outcome variable. Probability of and calculated risk for recurrence were determined by actuarial analysis. The criteria for statistical analysis were date of operation and date of death. Survival curves were compared by the log-rank test for various recurrence factors and Cox's model for multivariate analysis. A P-value of $<0.05$ was considered statistically significant. Statistical analyses were performed using the Prism 5.0 software (GraphPad Prism Software, La Jolla, CA, USA), and the
Statistical Package for Social Sciences for Windows (SPSS, Inc., Chicago, IL, USA).

\section{Results}

Cross-cancer mRNA expression and alterations in the YAPI gene. YAP1 mRNA expression in cases of OSC was higher than in 21 other cancer types recorded in the TCGA database. mRNA expression of YAP1 was lowest in acute myeloid leukemia (Fig. 1). Cross-cancer alteration was investigated in 21 types of cancer, and YAP1 expression in OSC was the greatest among the 21 types of cancers recorded in the TCGA.

YAP1 $m R N A$ expression in OSC. The present study examined YAP1 mRNA expression in OSC compared with 8 normal control samples (Fig. 2). Clinicopathological information of the patients is shown in Table I. YAP1 mRNA expression was significantly higher in cases of OSC compared to normal controls (Fig. 2A). YAP1 mRNA expression was higher in stages III and IV compared to earlier stages (Fig. 2B). When comparing YAP1 mRNA expression in 4 subtypes of ovarian cancer, differentiated, immunoreactive, mesenchymal and proliferative, and in 2 subtypes of ovarian cancer, integrated mesenchymal and epithelial subtypes $(23,24)$, YAP1 mRNA expression in the proliferative subtype was significantly higher than that in the differentiated, immunoreactive and mesenchymal subtypes (Fig. 2C). However, there was no significant difference in expression between the integrated mesenchymal subtype vs. the integrated epithelial subtype (Fig. 2D).

YAPl protein expression in OSC. When a comparison was conducted between stages of ovarian cancer, YAP1 protein expression was only significantly higher in stage IV compared to stages I, II and III (Fig. 3A). The proliferative and differentiated subtypes showed significantly higher protein expression than did the immunoreactive subtype (Fig. 3B). However, there was no significant difference in YAP1 protein level between the integrated epithelial and mesenchymal subtypes (Fig. 3C). The phosphorylated form of YAP1, at serine 127 (pYAP), which is inactivate and is localized to the cytoplasm, did not show any significant differences in protein expression (Fig. 3D). pYAP in the immunoreactive subtype was significantly lower than that in other subtypes; however, the pYAP/YAP ratio, which indicates higher YAP1 activity when it is lower, was lower in stage III than in stage I (Fig. 3E and G). There was no significant difference in the pYAP/YAP ratio between the subtypes of ovarian cancer (Fig. 3H and I).

GeneNeighbors of YAP1. The range of YAP1 mRNA expression in the 590 OSC samples was $2.12\left(\log _{2}\right)$ to 9.78 $\left(\log _{2}\right)$, with a fold-change of 4.61 . The 100 genes that were most highly correlated with YAP1 were selected using GeneNeighbors (Fig. 4A), and classified using DAVID. The genes were classified into 3 groups based on biological processes, cellular components and molecular functions. GO terms with significant differences $(\mathrm{P}<0.05)$ were: i) biological process, ii) cellular components, and iii) molecular functions. Genes highly expressed in OSC were mainly associated with the cell cycle (cell cycle process, cell cycle and cell cycle phase) and protein complexes (protein localization, protein complex 


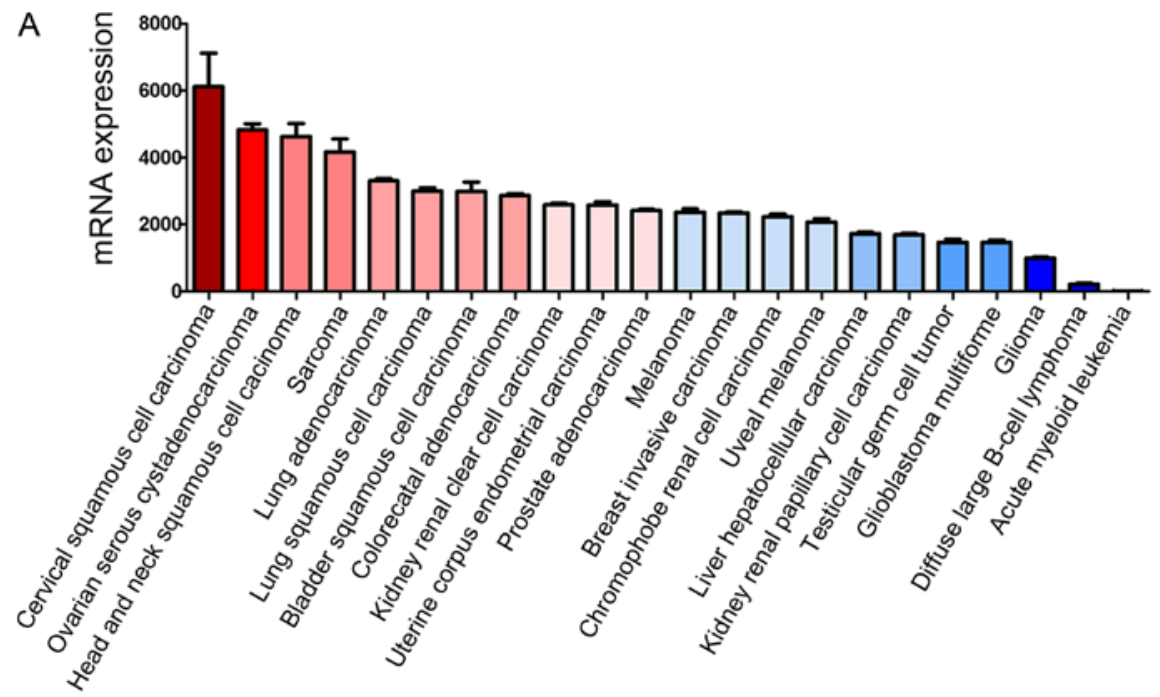

B

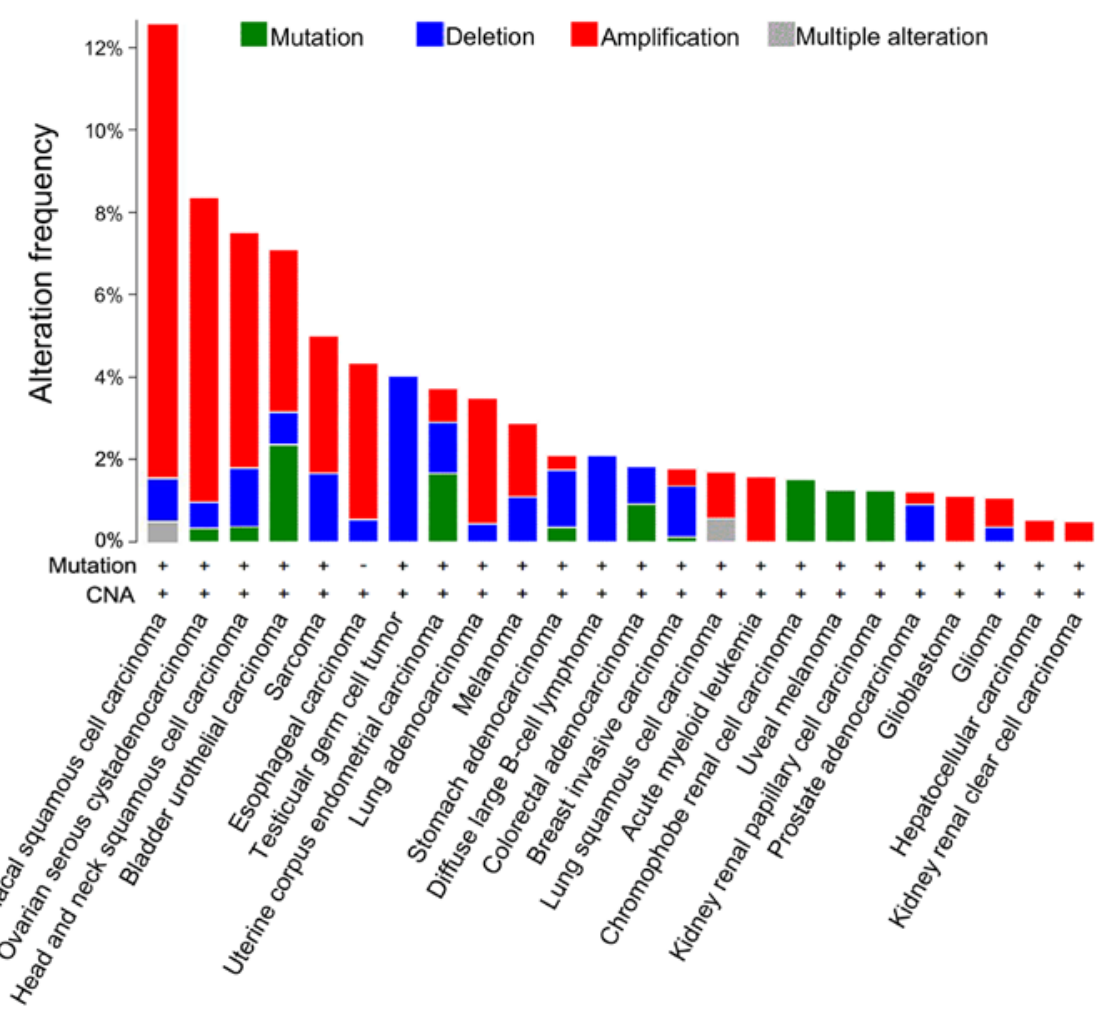

Figure 1. Cross-cancer mRNA expression of YAP1. (A) The data depict the mRNA expression of YAP1 in different cancer types based on the TCGA (https://tcga-data.nci.nih.gov/tcga/) data portal. (B) The data depict the frequency of alterations in YAP1 across different cancer types based on the TCGA. Potential alterations include mutations, deletions, amplification or multiple alterations. Data were obtained from the cBio database for cancer genomics (http:// cbioportal.org/public-portal/).

biogenesis and protein complex assembly) when analyzed by biological process (Fig. 4B). Genes highly expressed in OSC were mainly associated with the cytosol and ubiquitin ligase complexes when analyzed by cellular components. Genes highly expressed in OSC were mainly associated with ATP-dependent peptidase activity when analyzed by molecular function. In addition, when genes were analyzed according to cell signaling pathway [Kyoto Encyclopedia of Genes and Genomes (KEGG)], 5 signaling pathways had significant P-values. The analysis illustrated the importance of the ATM signaling pathway, the role of BRCA1, BRCA2 and
ATR in cancer susceptibility, the Cdc25 and Chk1 regulatory pathways that respond to DNA damage, regulation of cell cycle progression by Plk3, and RB tumor-suppressor/checkpoint signaling in response to DNA damage.

ClassNeighbors of YAP1 upregulated and downregulated in $O S C$. Analysis using ClassNeighbors yielded 2 classes of OSC: Class A contained the top 59 (10\%) YAP1-upregulated OSC samples and Class B contained the 59 (10\%) most YAP1-downregulated OSC samples (Fig. 5A). Of the 17,814 probe sets, the 200 genes that were most strongly 
Table I. Clinicopathological information of the ovarian serous cystadenocarcinoma patients of The Cancer Genome Atlas (TCGA).

\begin{tabular}{|c|c|c|c|c|c|c|c|c|c|}
\hline \multirow[b]{2}{*}{ Feature } & \multicolumn{3}{|c|}{ mRNA YAP expression } & \multicolumn{3}{|c|}{ YAP protein expression } & \multicolumn{3}{|c|}{$\begin{array}{l}\text { Phosphorylated YAP } \\
\text { protein expression }\end{array}$} \\
\hline & Total & 2X Down & $2 X U p$ & Low & Intermediate & High & Low & Intermediate & High \\
\hline No. of patients & 563 & 205 & 83 & 137 & 138 & 137 & 137 & 138 & 137 \\
\hline Mean age (years) & 59.7 & 60.2 & 58.8 & 61.1 & 59.7 & 61.3 & 61.7 & 58.5 & 58.9 \\
\hline \multicolumn{10}{|l|}{ Stage } \\
\hline I & 16 & 9 & 0 & 5 & 3 & 3 & 3 & 9 & 2 \\
\hline II & 27 & 11 & 4 & 6 & 7 & 8 & 10 & 4 & 7 \\
\hline III & 440 & 152 & 66 & 108 & 105 & 110 & 110 & 109 & 103 \\
\hline IV & 85 & 30 & 13 & 16 & 22 & 16 & 14 & 14 & 23 \\
\hline \multicolumn{10}{|l|}{ Tumor grade } \\
\hline G1 & 6 & 4 & 0 & 1 & 0 & 2 & 2 & 2 & 1 \\
\hline G2 & 65 & 29 & 7 & 15 & 20 & 16 & 15 & 17 & 22 \\
\hline G3 & 478 & 166 & 75 & 112 & 117 & 118 & 117 & 116 & 113 \\
\hline \multicolumn{10}{|l|}{ Surgical outcome } \\
\hline Optimal & 369 & 125 & 55 & 86 & 87 & 91 & 85 & 86 & 88 \\
\hline Suboptimal & 142 & 56 & 17 & 30 & 38 & 36 & 39 & 36 & 37 \\
\hline \multicolumn{10}{|l|}{ Vital status } \\
\hline Living & 269 & 100 & 37 & 60 & 65 & 61 & 62 & 66 & 67 \\
\hline Deceased & 291 & 103 & 45 & 76 & 73 & 75 & 75 & 71 & 68 \\
\hline
\end{tabular}

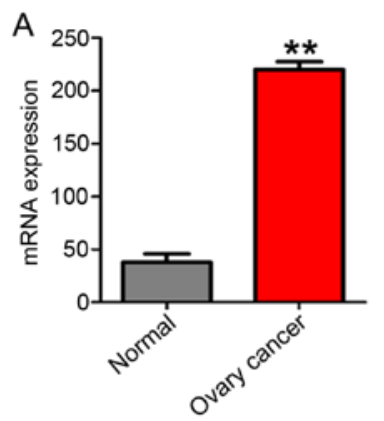

C

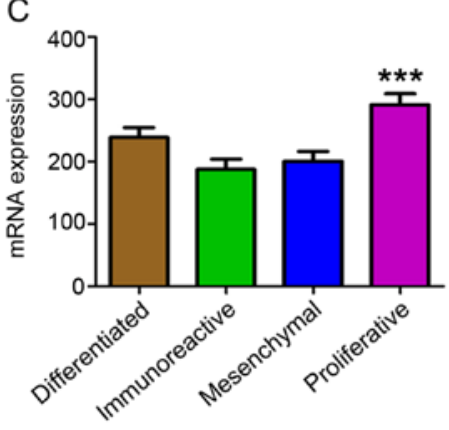

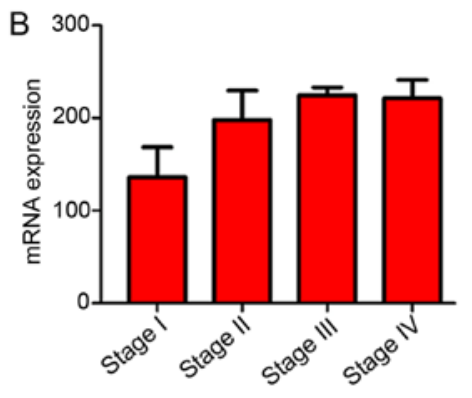

$\mathrm{D}$

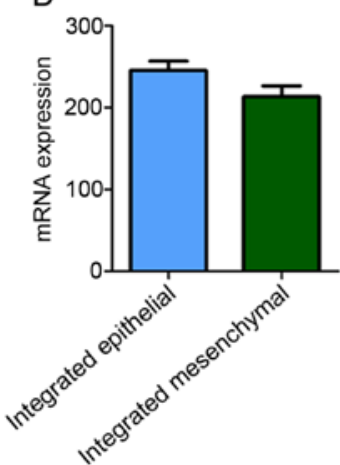

Figure 2. (A-D) YAP1 mRNA expression in ovarian serous adenocarcinonoma. mRNA microarray data of YAP1 in normal controls and ovarian serous cystadenocarcinoma patients, obtained from the TCGA data portal (https://tcga-data.nci.nih.gov/tcga/). mRNA microarray data of YAP1 in various cell types of epithelial ovarian carcinoma, obtained from the CCLE data portal (http://www.broadinstitute.org/ccle/); ${ }^{* *} \mathrm{P}<0.01$ and ${ }^{* * *} \mathrm{P}<0.001$. One way ANOVA was performed for comparisons between more than 2 groups, and t-tests were performed for comparisons between 2 groups.

correlated and most highly expressed in Classes A and B were selected. DAVID analysis classified these genes into groups based on GO terms: i) biological processes, ii) cellular components, iii) molecular functions, and iv) the KEGG 

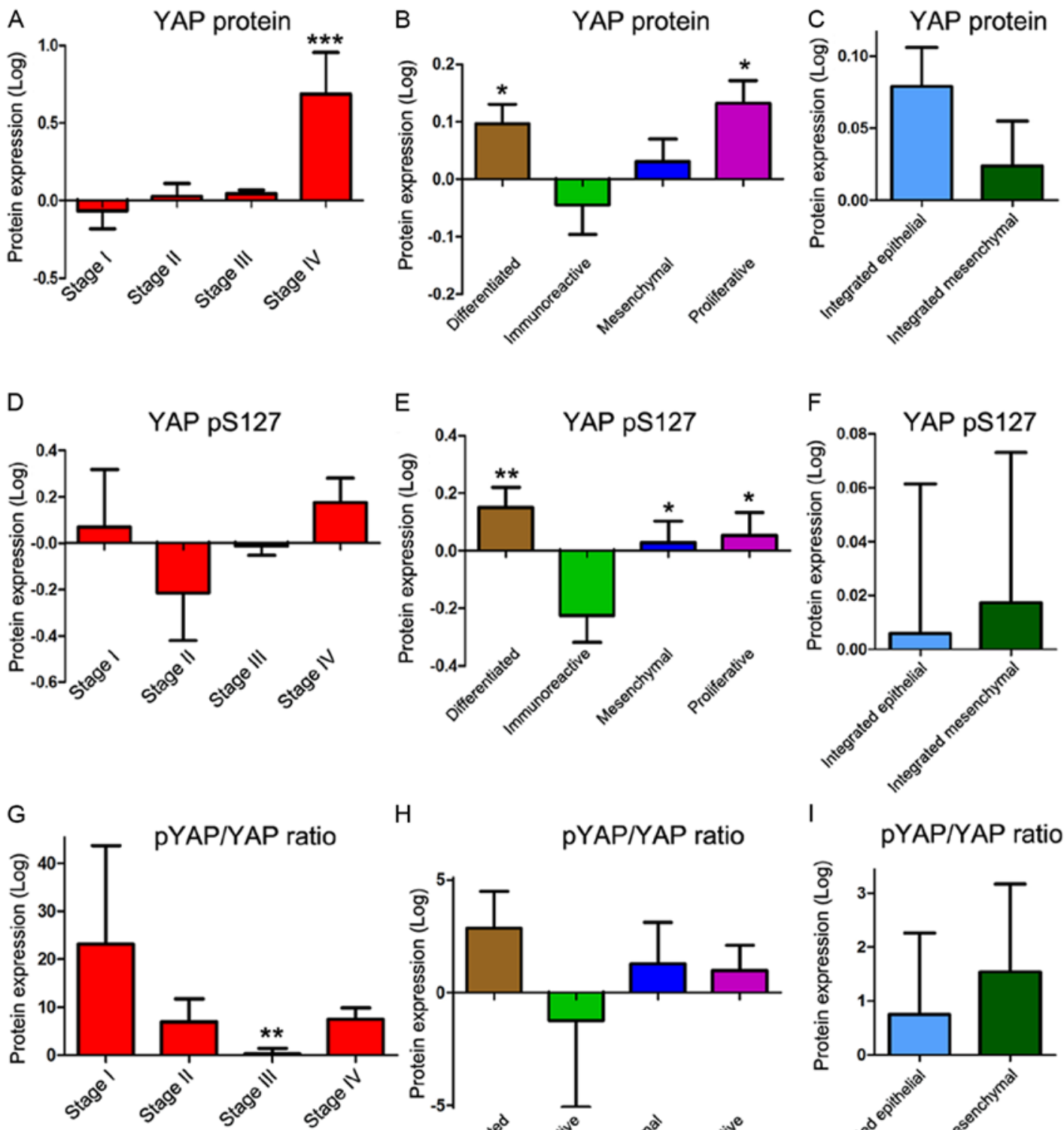

$\mathrm{H}$
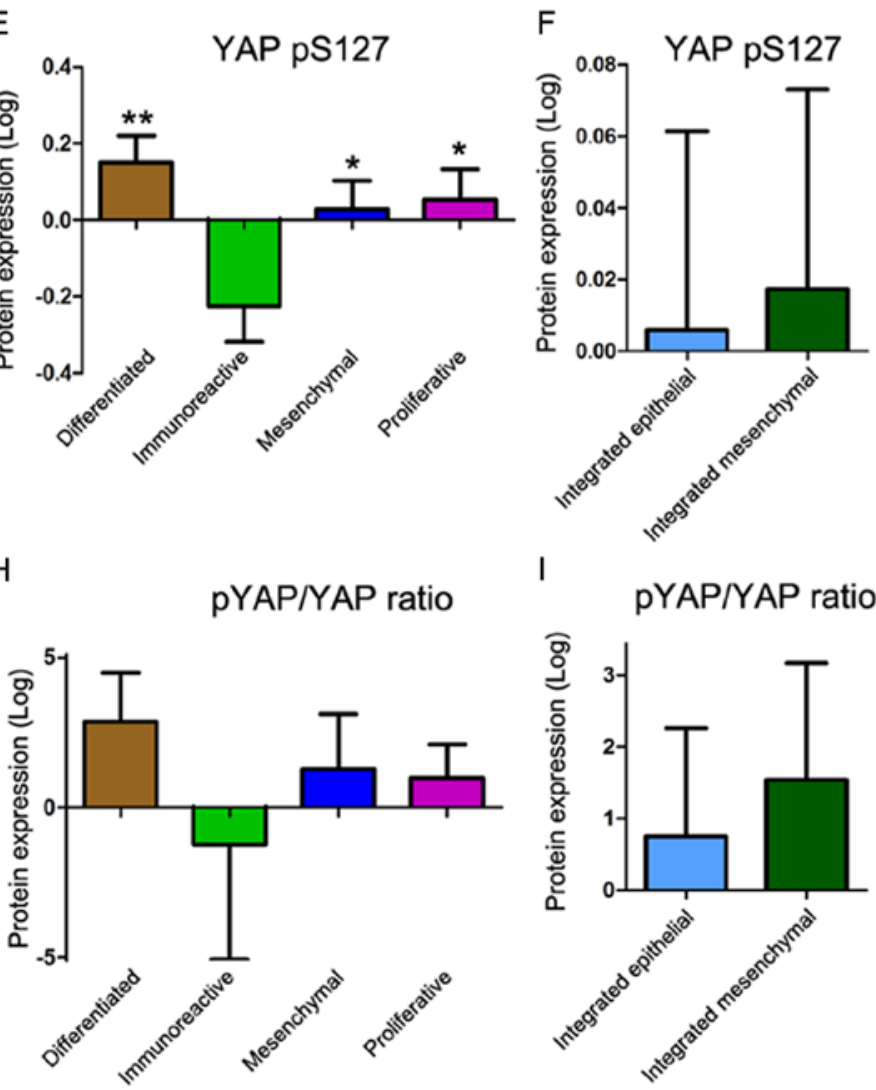

1

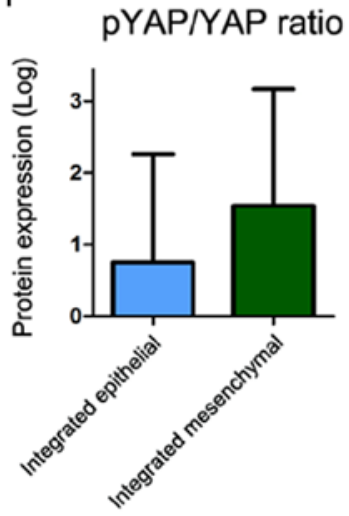

Figure 3. (A-I) YAP1 protein expression in ovarian serous adenocarcinonoma. Protein expression data of YAP1 in ovarian serous cystadenocarcinoma, obtained from the TCGA data portal (https://tcga-data.nci.nih.gov/tcga/); ${ }^{*} \mathrm{P}<0.05,{ }^{* *} \mathrm{P}<0.01$ and ${ }^{* * *} \mathrm{P}<0.001$. One way ANOVA was performed for comparisons between more than 2 groups, and t-tests were performed for comparisons between 2 groups.

pathway (Fig. 5B and C and Table II). Genes highly expressed in Class A were mostly associated with DNA recombination and the cell cycle (biological processes), intracellular organelle lumen (cellular components), and RNA and nucleotide binding (molecular functions) (Fig. 5B). Genes highly expressed in Class B were mostly associated with nucleosome and chromatin assembly (biological processes), nucleosomes and the respiratory chain (cellular components), and NADH dehydrogenase (molecular functions) (Fig. 5C).

In addition, GSEA was performed in order to investigate the significantly enriched pathways that differed between Classes A and B. In Class A, pathways involving tight junctions, endometrial cancer, WNT signaling, TGF- $\beta$ signaling, adherent junctions, basal cell carcinoma and prostate cancer were significantly enriched when compared with Class B. In
Class B, pathways involved with primary immunodeficiency, systematic lupus erythematosus, the intestinal immune network for IgA production, regulation of autophagy, autoimmune thyroid disease and natural killer cell-mediated cytotoxicity were enriched (Table III). In Class A, WNT (25) and TGF- $\beta$ signaling (26) were related to cancer progression (Fig. 6A). Immune-related signaling pathways were related to Class B (Fig. 6B).

Survival analysis. In order to determine the prognostic significance of YAP1 expression in patients with OSC, we assessed the correlation between YAP mRNA and protein expression profiles and clinically significant characteristics: survival, tumor stage, grade and residual disease status. Initially, Kaplan-Meier curves were used to plot overall 


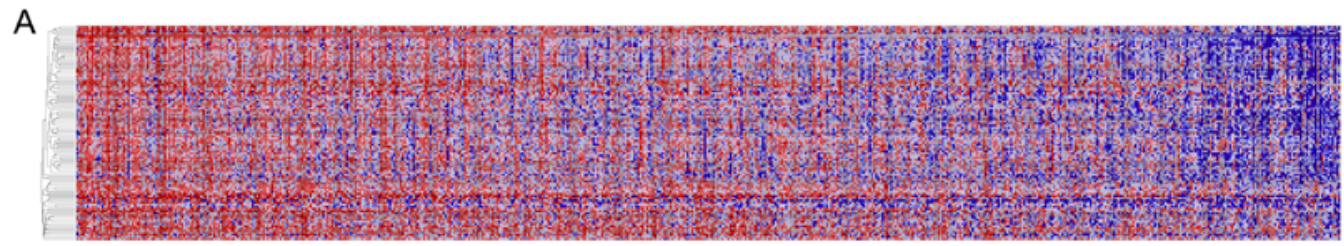

B

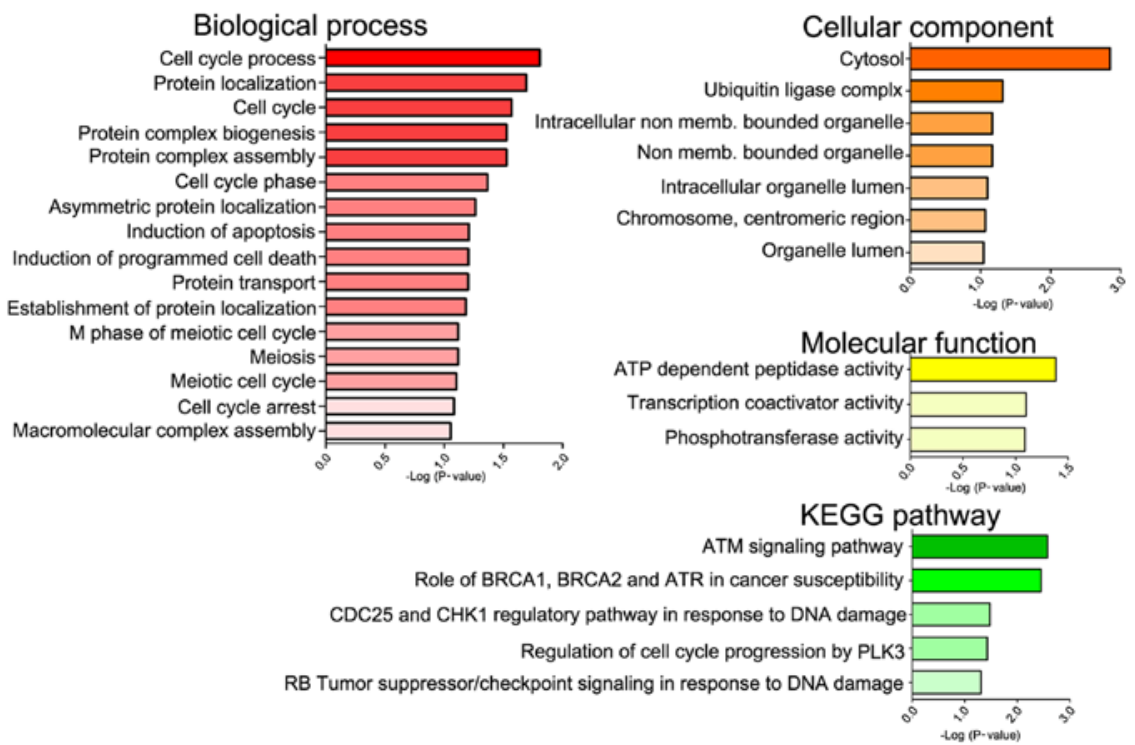

Figure 4. GeneNeighbors of YAP1 in 590 ovarian serous cystadenocarcinoma samples. Hierarchical clustering of YAP1 GeneNeighbors in ovarian serous cystadenocarcinoma. Ovarian serous cystadenocarcinoma samples are arranged in decreasing order of YAP mRNA expression. Colors in the heat map represent expression relative to the mean expression value, with red indicating higher expression and blue indicating lower expression. (A) GeneNeighbors of YAP1 are shown in the column. (B) GeneNeighbors were characterized as biological processes, cellular components, molecular function and KEGG pathway-related.

A Class A

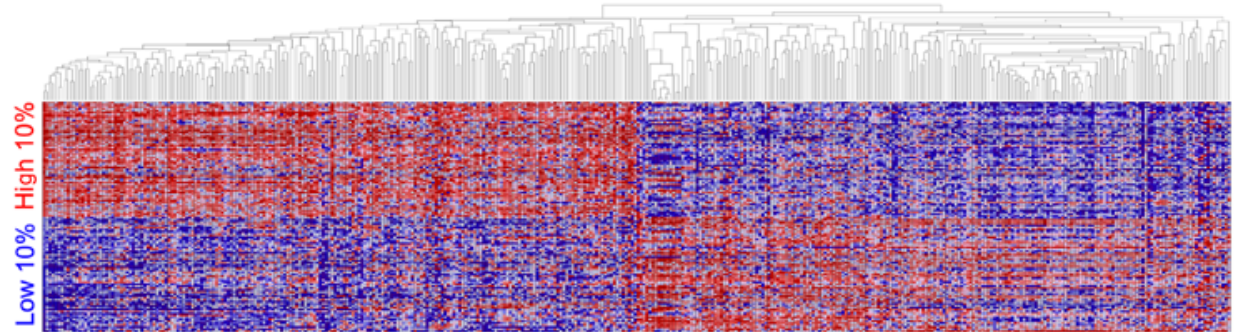

B

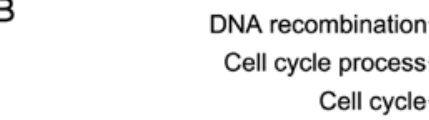

Cellular marcromol. catabolic process

BMP signaling pathway Intracellular organelle lumen-CC

Organelle lumen

Membrane enclosed lumen

Mitochondrial lument

Mitochondrial matrix

RNA binding-N

Nucleotide binding

Intramolecular transferase activity

Protein homodimerization activity

ATPase activity

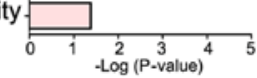

C

Nucleosome assembly- $\mathrm{BP}$

Chromatin assembly

Cellular macromolar complex

Protein-DNA complex assembly.

Nucleosome organization

Nucleosome-CC

Respiratoy chain

Protein-DNA complex

Mitochondrial respiratory chain-

Mitochondrial part

NADH dehydrogenase activity-MF

NADH dehydrogenase (ubiquinone) activity.

NADH dehydrogenase (quinone) activity.

Potassium channel activity

Oxidoreductase activity

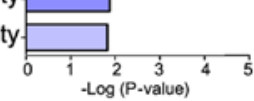

Figure 5. ClassNeighbors of YAP1-related genes in 2 classes of ovarian serous cystadenocarcinoma samples. Hierarchical clustering of differentially expressed genes (top 10\%) upregulated and downregulated in OSC cases according to Pearson distance. (A) Colors in the heat map represent expression relative to the mean expression value, with red indicating higher expression and blue indicating lower expression. (B and C) Genes in classes A and B were divided into biological processes, cellular components and molecular functions. 
Table II. DAVID analysis of ClassNeighbors.

A, Class A

Biological process (BP)

GO:0006310 DNA recombination

GO:0022402 cell cycle process

GO:0007049 cell cycle

GO:0044265 cellular macromolecule catabolic process

9.19

0.007

GO:0030509 BMP signaling pathway

8.65

0.009

GO:0008104 protein localization

2.16

0.011

GO:0022403 cell cycle phase

9.73

0.011

GO:0000077 DNA damage checkpoint

5.95

0.012

GO:0009451 RNA modification

GO:0000075 cell cycle checkpoint

GO:0009057 macromolecule catabolic process

GO:0031570 DNA integrity checkpoint

GO:0007126 meiosis

GO:0051327 M phase of meiotic cell cycle

GO:0010719 negative regulation of epithelial to mesenchymal transition

GO:0051321 meiotic cell cycle

GO:0065003 macromolecular complex assembly

GO:0007178 transmembrane receptor protein serine/threonine kinase signaling pathway

GO:0007131 reciprocal meiotic recombination

GO:0045596 negative regulation of cell differentiation

GO:0015031 protein transport

GO:0010771 negative regulation of cell morphogenesis involved in differentiation

2.16

0.014

2.16

0.014

2.70

0.015

0.017

2.16

0.017

2.70

0.020

GO:0045184 establishment of protein localization

GO:0051276 chromosome organization

GO:0051222 positive regulation of protein transport

GO:0050821 protein stabilization

GO:0043933 macromolecular complex subunit organization

GO:0016567 protein ubiquitination

GO:0002377 immunoglobulin production

GO:0016071 mRNA metabolic process

GO:0002440 production of molecular mediator of immune response

GO:0006974 response to DNA damage stimulus

GO:0032446 protein modification by small protein conjugation

15

11

Cellular component (CC)

GO:0070013 intracellular organelle lumen

GO:0043233 organelle lumen

GO:0031974 membrane-enclosed lumen

GO:0031980 mitochondrial lumen

GO:0005759 mitochondrial matrix

GO:0000794 condensed nuclear chromosome

GO:0000793 condensed chromosome

GO:0005829 cytosol

GO:0031981 nuclear lumen

GO:0030135 coated vesicle

GO:0000228 nuclear chromosome

GO:0044429 mitochondrial part

GO:0005694 chromosome
1.08

0.031

8.11

0.031

5.95

0.032

2.16

0.033

1.62

0.035

7.57

0.036

2.70

0.037

1.62

0.039

4.86

0.041

1.62

0.042

4.86

0.043

2.70

0.050

17.84

0.000

17.84

0.000

17.84

0.000

5.41

0.000

5.41

0.000

2.70

0.001

3.24

0.007

11.89

0.009

12.43

0.012

3.24

0.015

3.24

0.017

6.49

0.020

5.41

0.025 
Table II. Continued.

A, Class A

\begin{tabular}{|c|c|c|c|}
\hline Term & Count & $\%$ & P-value \\
\hline GO:0005654 nucleoplasm & 15 & 8.11 & 0.030 \\
\hline GO:0042645 mitochondrial nucleoid & 3 & 1.62 & 0.033 \\
\hline GO:0009295 nucleoid & 3 & 1.62 & 0.033 \\
\hline GO:0031090 organelle membrane & 17 & 9.19 & 0.041 \\
\hline GO:0042175 nuclear envelope-endoplasmic reticulum network & 7 & 3.78 & 0.046 \\
\hline \multicolumn{4}{|l|}{ Molecular function (MF) } \\
\hline GO:0003723 RNA binding & 18 & 9.73 & 0.000 \\
\hline GO:0000166 nucleotide binding & 33 & 17.84 & 0.011 \\
\hline GO:0016866 intramolecular transferase activity & 3 & 1.62 & 0.025 \\
\hline GO:0042803 protein homodimerization activity & 8 & 4.32 & 0.041 \\
\hline GO:0016887 ATPase activity & 8 & 4.32 & 0.041 \\
\hline GO:0019237 centromeric DNA binding & 2 & 1.08 & 0.047 \\
\hline
\end{tabular}

B, Class B

\begin{tabular}{|c|c|c|c|}
\hline Term & Count & $\%$ & P-value \\
\hline \multicolumn{4}{|l|}{ Biological process (BP) } \\
\hline GO:0006334 nucleosome assembly & 7 & 3.91 & 0.000 \\
\hline GO:0031497 chromatin assembly & 7 & 3.91 & 0.000 \\
\hline GO:0034621 cellular macromolecular complex subunit organization & 13 & 7.26 & 0.000 \\
\hline GO:0065004 protein-DNA complex assembly & 7 & 3.91 & 0.000 \\
\hline GO:0034728 nucleosome organization & 7 & 3.91 & 0.000 \\
\hline GO:0006091 generation of precursor metabolites and energy & 12 & 6.70 & 0.000 \\
\hline GO:0022900 electron transport chain & 7 & 3.91 & 0.001 \\
\hline GO:0006323 DNA packaging & 7 & 3.91 & 0.001 \\
\hline GO:0034622 cellular macromolecular complex assembly & 11 & 6.15 & 0.002 \\
\hline GO:0006812 cation transport & 15 & 8.38 & 0.002 \\
\hline GO:0006333 chromatin assembly or disassembly & 7 & 3.91 & 0.002 \\
\hline GO:0006119 oxidative phosphorylation & 6 & 3.35 & 0.004 \\
\hline GO:0045454 cell redox homeostasis & 5 & 2.79 & 0.004 \\
\hline GO:0006811 ion transport & 17 & 9.50 & 0.006 \\
\hline GO:0043281 regulation of caspase activity & 5 & 2.79 & 0.009 \\
\hline GO:0006120 mitochondrial electron transport, NADH to ubiquinone & 4 & 2.23 & 0.009 \\
\hline GO:0052548 regulation of endopeptidase activity & 5 & 2.79 & 0.011 \\
\hline GO:0052547 regulation of peptidase activity & 5 & 2.79 & 0.012 \\
\hline GO:0015672 monovalent inorganic cation transport & 9 & 5.03 & 0.018 \\
\hline GO:0006917 induction of apoptosis & 9 & 5.03 & 0.019 \\
\hline GO:0012502 induction of programmed cell death & 9 & 5.03 & 0.019 \\
\hline GO:0042981 regulation of apoptosis & 16 & 8.94 & 0.020 \\
\hline GO:0042775 mitochondrial ATP synthesis coupled electron transport & 4 & 2.23 & 0.021 \\
\hline GO:0042773 ATP synthesis coupled electron transport & 4 & 2.23 & 0.021 \\
\hline GO:0043067 regulation of programmed cell death & 16 & 8.94 & 0.022 \\
\hline GO:0010941 regulation of cell death & 16 & 8.94 & 0.023 \\
\hline GO:0030001 metal ion transport & 11 & 6.15 & 0.024 \\
\hline GO:0051336 regulation of hydrolase activity & 9 & 5.03 & 0.025 \\
\hline GO:0006813 potassium ion transport & 6 & 3.35 & 0.026 \\
\hline GO:0022904 respiratory electron transport chain & 4 & 2.23 & 0.029 \\
\hline GO:0043933 macromolecular complex subunit organization & 14 & 7.82 & 0.034 \\
\hline GO:0042127 regulation of cell proliferation & 15 & 8.38 & 0.035 \\
\hline
\end{tabular}


Table II. Continued.

B, Class B

\begin{tabular}{|c|c|c|c|}
\hline Term & Count & $\%$ & P-value \\
\hline GO:0008285 negative regulation of cell proliferation & 9 & 5.03 & 0.035 \\
\hline GO:0043065 positive regulation of apoptosis & 10 & 5.59 & 0.036 \\
\hline GO:0007268 synaptic transmission & 8 & 4.47 & 0.037 \\
\hline GO:0043068 positive regulation of programmed cell death & 10 & 5.59 & 0.037 \\
\hline GO:0010942 positive regulation of cell death & 10 & 5.59 & 0.038 \\
\hline GO:0050728 negative regulation of inflammatory response & 3 & 1.68 & 0.041 \\
\hline GO:0044093 positive regulation of molecular function & 12 & 6.70 & 0.043 \\
\hline GO:0006325 chromatin organization & 9 & 5.03 & 0.044 \\
\hline GO:0050727 regulation of inflammatory response & 4 & 2.23 & 0.045 \\
\hline \multicolumn{4}{|l|}{ Cellular component (CC) } \\
\hline GO:0000786 nucleosome & 7 & 3.91 & 0.000 \\
\hline GO:0070469 respiratory chain & 7 & 3.91 & 0.000 \\
\hline GO:0032993 protein-DNA complex & 7 & 3.91 & 0.000 \\
\hline GO:0005746 mitochondrial respiratory chain & 6 & 3.35 & 0.000 \\
\hline GO:0044429 mitochondrial part & 16 & 8.94 & 0.001 \\
\hline GO:0044455 mitochondrial membrane part & 7 & 3.91 & 0.002 \\
\hline GO:0019866 organelle inner membrane & 11 & 6.15 & 0.002 \\
\hline GO:0005739 mitochondrion & 22 & 12.29 & 0.002 \\
\hline GO:0005740 mitochondrial envelope & 12 & 6.70 & 0.003 \\
\hline GO:0005743 mitochondrial inner membrane & 10 & 5.59 & 0.003 \\
\hline GO:0000785 chromatin & 8 & 4.47 & 0.004 \\
\hline GO:0031966 mitochondrial membrane & 11 & 6.15 & 0.006 \\
\hline GO:0009897 external side of plasma membrane & 7 & 3.91 & 0.007 \\
\hline GO:0045271 respiratory chain complex I & 4 & 2.23 & 0.008 \\
\hline GO:0005747 mitochondrial respiratory chain complex I & 4 & 2.23 & 0.008 \\
\hline GO:0030964 NADH dehydrogenase complex & 4 & 2.23 & 0.008 \\
\hline GO:0031967 organelle envelope & 14 & 7.82 & 0.009 \\
\hline GO:0031975 envelope & 14 & 7.82 & 0.009 \\
\hline GO:0009986 cell surface & 9 & 5.03 & 0.023 \\
\hline GO:0031090 organelle membrane & 19 & 10.61 & 0.023 \\
\hline GO:0044427 chromosomal part & 9 & 5.03 & 0.039 \\
\hline \multicolumn{4}{|l|}{ Molecular function (MF) } \\
\hline GO:0003954 NADH dehydrogenase activity & 4 & 2.23 & 0.010 \\
\hline GO:0008137 NADH dehydrogenase (ubiquinone) activity & 4 & 2.23 & 0.010 \\
\hline GO:0050136 NADH dehydrogenase (quinone) activity & 4 & 2.23 & 0.010 \\
\hline GO:0005267 potassium channel activity & 6 & 3.35 & 0.013 \\
\hline $\begin{array}{c}\text { GO:0016655 oxidoreductase activity, acting on NADH or NADPH, } \\
\text { quinone or similar compound as acceptor }\end{array}$ & 4 & 2.23 & 0.015 \\
\hline GO:0047485 protein N-terminus binding & 4 & 2.23 & 0.043 \\
\hline GO:0030955 potassium ion binding & 5 & 2.79 & 0.047 \\
\hline
\end{tabular}

survival in samples with mRNA expression that was either 2-fold upregulated or downregulated (Fig. 7). YAP1 mRNA expression was not significantly associated with patient prognosis in OSC (Fig. 7A). To determine whether YAP and pYAP distribution are associated with overall patient survival in OSC, YAP and pYAP expression levels were categorized as high, intermediate and low, since neither
YAP nor pYAP alone were associated with OSC prognosis. Among 9 categories studied, the category of high YAP and low pYAP showed the poorest prognosis (Fig. 7B). The category of high YAP and low pYAP showed significantly poorer prognosis than did the category of high YAP and high pYAP and the category of intermediate YAP and intermediate pYAP (Fig. 7C and D). 
A

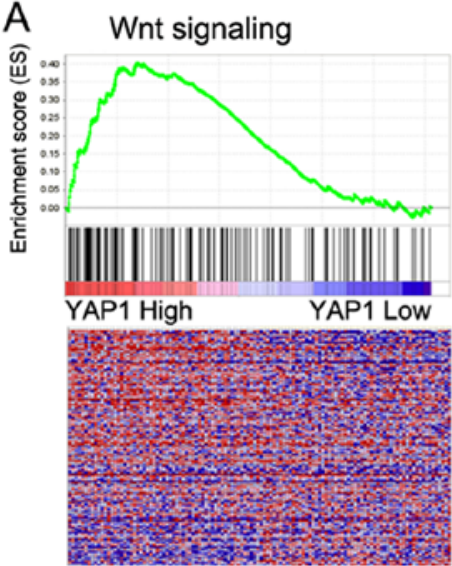

Normal p-value $\quad 0.019$

FDR q-value $\quad 0.34$

FWER p-value $\quad 0.67$

\begin{tabular}{lccc}
$\begin{array}{l}\text { Gene } \\
\text { symbol }\end{array}$ & $\begin{array}{c}\text { Rank in } \\
\text { gene list }\end{array}$ & $\begin{array}{c}\text { Rank } \\
\text { metric score }\end{array}$ & $\begin{array}{c}\text { Running } \\
\text { ES }\end{array}$ \\
\hline DKK4 & 3540 & 0.146 & 0.403 \\
PPP2R1A & 3453 & 0.150 & 0.402 \\
RHOA & 3427 & 0.151 & 0.397 \\
NKD1 & 3387 & 0.153 & 0.392 \\
WNT71 & 2821 & 0.178 & 0.391 \\
FRAT2 & 2720 & 0.182 & 0.389 \\
TP53 & 2607 & 0.187 & 0.388 \\
TBL1XR1 & 3362 & 0.154 & 0.387 \\
DVL1 & 3331 & 0.155 & 0.382 \\
WNT10A & 2595 & 0.188 & 0.380
\end{tabular}

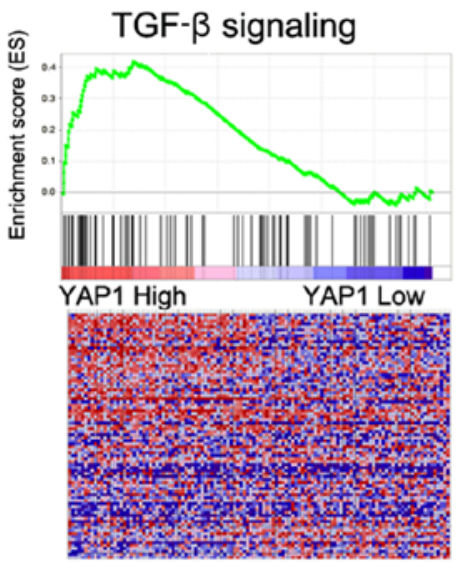

Normal p-value $\quad 0.028$

FDR q-value $\quad 0.29$

FWER p-value

0.80

$\begin{array}{lll}\text { Gene } & \text { Rank in } \\ \text { symbol Rank } & \text { Running }\end{array}$

\begin{tabular}{llll}
\hline PPP2R1A & 3453 & 0.150 & 0.421 \\
RHOA & 3427 & 0.151 & 0.412 \\
BMP4 & 3383 & 0.153 & 0.405 \\
ID4 & 3255 & 0.158 & 0.402 \\
PPP2R1B & 1650 & 0.241 & 0.398 \\
SMAD2 & 3240 & 0.158 & 0.392 \\
SMAD3 & 2025 & 0.218 & 0.391 \\
ROCK1 & 2500 & 0.192 & 0.390 \\
TGFBR2 & 3176 & 0.162 & 0.385 \\
SMAD4 & 1609 & 0.244 & 0.385
\end{tabular}

B

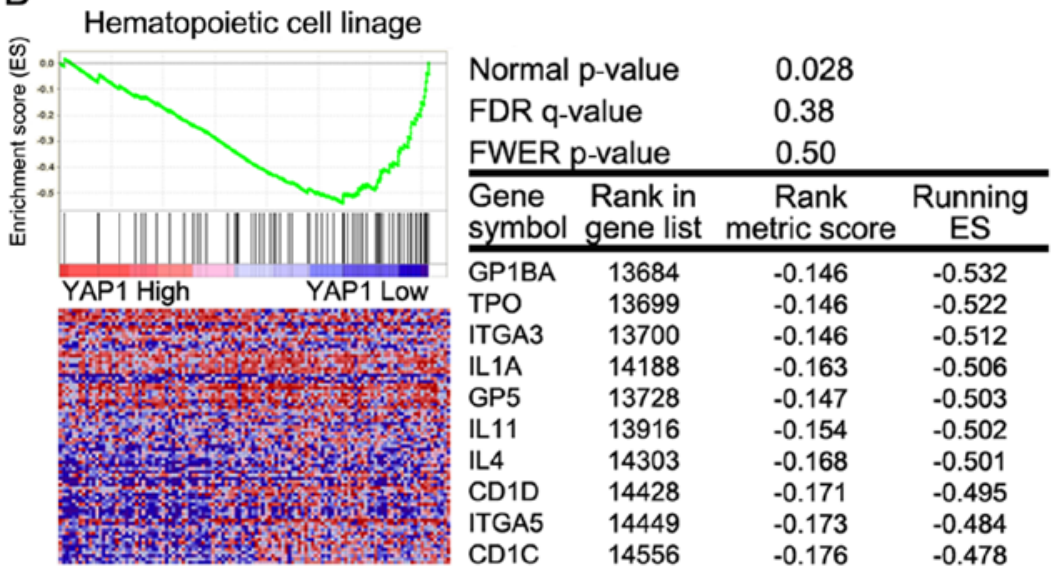

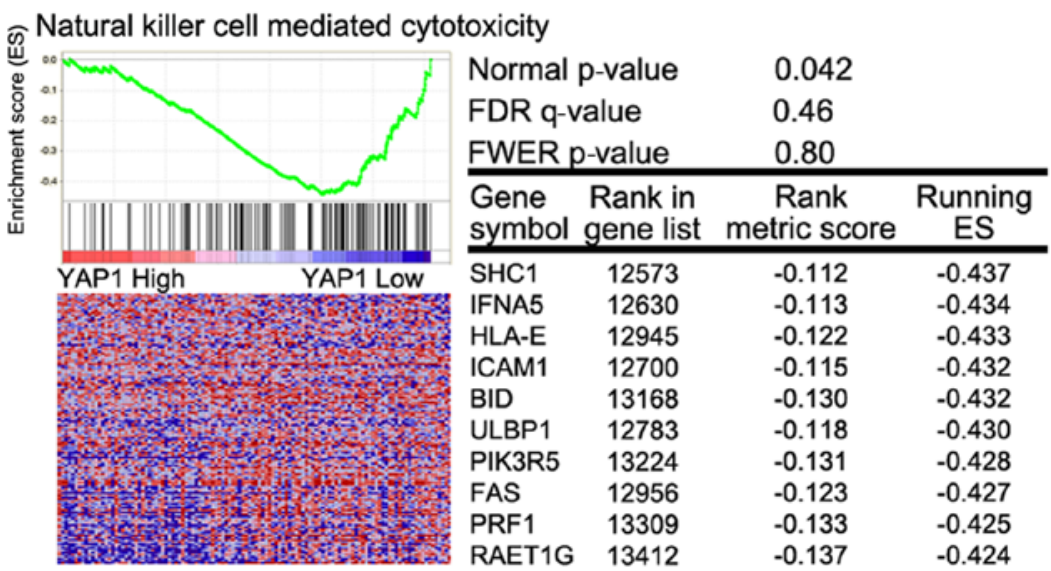

Figure 6. (A) GSEA analysis of Class A and B. WNT and TGF- $\beta$ signaling were significantly enriched in Class A. (B) Hematopoietic cell lineage pathway and natural killer mediated cytotoxicity pathway were significantly enriched in Class B. 
Table III. Gene set enrichment analysis (GSEA) of Class A and Class B.

A, Class A

\begin{tabular}{|c|c|c|c|c|}
\hline Name & Size & ES & NES & NOM p-val \\
\hline KEGG_TIGHT_JUNCTION & 125 & 0.38 & 1.63 & 0.004 \\
\hline KEGG_ENDOMETRIAL_CANCER & 52 & 0.49 & 1.67 & 0.014 \\
\hline KEGG_WNT_SIGNALING_PATHWAY & 147 & 0.40 & 1.63 & 0.019 \\
\hline KEGG_SELENOAMINO_ACID_METABOLISM & 23 & 0.55 & 1.62 & 0.025 \\
\hline KEGG_LYSINE_DEGRADATION & 43 & 0.49 & 1.64 & 0.025 \\
\hline KEGG_AMINOACYL_TRNA_BIOSYNTHESIS & 41 & 0.54 & 1.60 & 0.026 \\
\hline KEGG_TGF_BETA_SIGNALING_PATHWAY & 82 & 0.42 & 1.57 & 0.028 \\
\hline KEGG_ADHERENS_JUNCTION & 73 & 0.46 & 1.62 & 0.032 \\
\hline KEGG_BASAL_CELL_CARCINOMA & 55 & 0.51 & 1.69 & 0.036 \\
\hline KEGG_PROSTATE_CANCER & 87 & 0.37 & 1.48 & 0.049 \\
\hline
\end{tabular}

B, Class B

\begin{tabular}{|c|c|c|c|c|}
\hline KEGG_ARACHIDONIC_ACID_METABOLISM & 51 & -0.43 & -1.58 & 0.010 \\
\hline KEGG_PRIMARY_IMMUNODEFICIENCY & 34 & -0.61 & -1.73 & 0.026 \\
\hline KEGG_SYSTEMIC_LUPUS_ERYTHEMATOSUS & 114 & -0.61 & -1.86 & 0.027 \\
\hline KEGG_HEMATOPOIETIC_CELL_LINEAGE & 79 & -0.54 & -1.71 & 0.029 \\
\hline KEGG_ALPHA_LINOLENIC_ACID_METABOLISM & 17 & -0.54 & -1.53 & 0.034 \\
\hline $\begin{array}{l}\text { KEGG_INTESTINAL_IMMUNE_NETWORK_FOR_ } \\
\text { IGA_PRODUCTION }\end{array}$ & 43 & -0.51 & -1.60 & 0.038 \\
\hline KEGG_REGULATION_OF_AUTOPHAGY & 32 & -0.44 & -1.51 & 0.039 \\
\hline KEGG_AUTOIMMUNE_THYROID_DISEASE & 47 & -0.54 & -1.62 & 0.042 \\
\hline KEGG_NATURAL_KILLER_CELL_MEDIATED_ & & & & \\
\hline CYTOTOXICITY & 128 & -0.44 & -1.57 & 0.043 \\
\hline
\end{tabular}

ES, enrichment score; NES, normalized enrichment score; NOM p-val, norminal p-value.
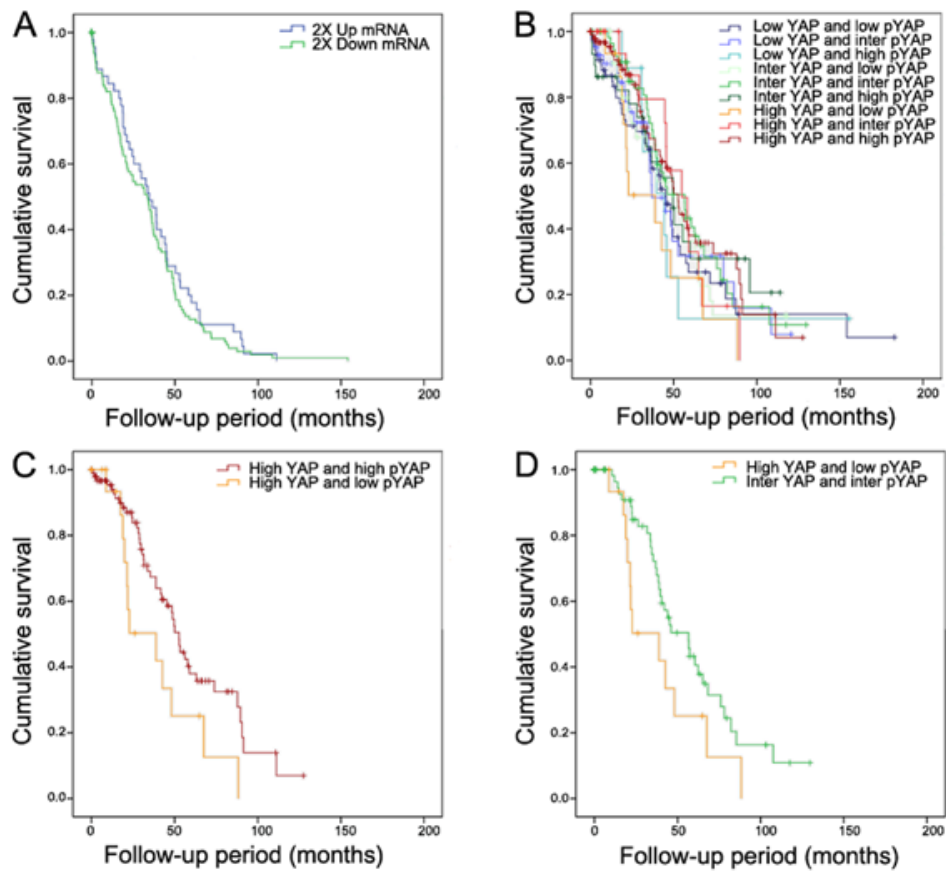

Figure 7. Survival analysis. High YAP and low pYAP protein expression were correlated with poor prognosis. Kaplan-Meier analysis of the association between YAP mRNA and protein expression, and overall survival. (A) Kaplan-Meier curves were used to plot overall survival with mRNA expression. YAP and pYAP expression levels were categorized as high, intermediate and low. (B) Among 9 categories, the category of high YAP and low pYAP showed the poorest prognosis. (C) $\mathrm{P}=0.042$. (D) $\mathrm{P}=0.065$. $\mathrm{P}$-value was determined by log-rank tests. 


\section{Discussion}

In the present study, alterations in the YAP1 gene in cases of OSC were found to be higher than that in various other cancer types. YAP1 mRNA expression was significantly higher in OSC compared with normal ovarian samples, and was higher in stages III and IV than in stages I and II. YAP1 protein, which mainly localized to the nucleus, was also expressed more highly in stage IV than in stages I ,II and III. However, the protein level of pYAP1, which is localized to the cytoplasm, was not significantly different between stages. The ratio of pYAP/YAP, which indicates higher activity at a low ratio, was lower in stage III than in stages I and II. When considering OSC subtypes, YAP1 mRNA and protein expression in the proliferative subtype was significantly higher than that in the differentiated, immunoreactive and mesenchymal subtypes. However, there was no significant difference in YAP1 mRNA or protein expression between the integrated mesenchymal and the integrated epithelial subtypes. In bioinformatic analysis, YAP1 was mainly correlated with the cell cycle. TGF- $\beta$ and WNT signaling were significantly increased in the high-YAP1 class as assessed by gene set enrichment analysis. Finally, high-YAP and low-pYAP were associated with poor overall survival in cases of OSC.

Elevated YAP1 expression and nuclear localization have been observed in multiple types of human cancers, including liver, colon, lung and prostate cancer $(6-8,27)$. In hepatocellular carcinoma, YAP1 was found to be an independent prognostic marker for overall and disease-free survival (13). In epithelial ovarian cancer, subcellular levels of YAP1 showed an exceptionally strong association with poor prognosis; high levels of nuclear YAP or low levels of cytoplasmic phosphorylated YAP1 were associated with poor prognosis (28). Patients with both high levels of nuclear YAP and low levels of phosphorylated YAP had an 50\% lower 5-year survival rate, and this combination served as an independent prognostic marker for survival (28). In accordance with previous findings, we showed that high YAP and low pYAP were associated with a poor prognosis. High YAP1 expression and its subcellular distribution may be related to poor overall survival in OSC. This finding should be confirmed in further studies.

The Cancer Genome Atlas Research Network separates OSC into 4 subtypes (immunoreactive, differentiated, proliferative and mesenchymal) based on mRNA analysis (24). Yang et al found that the integrated epithelial and mesenchymal subtypes were associated with poor overall survival based on miRNA analysis of OSC patients (23). In the present study, we revealed that YAP1 mRNA and protein expression in the proliferative subtype was significantly higher than that in the differentiated, immunoreactive and mesenchymal subtypes. However, there was no significant difference in YAP1 mRNA and protein expression between the integrated mesenchymal subtype and the integrated epithelial subtype. Molecular subgroups of ovarian cancer have been poorly examined and need to be further elucidated.

To verify the involvement of YAP1 in OSC, we performed bioinformatic analysis. This analysis revealed that cell cycle- and protein localization-related genes were highly correlated with YAP1 in 563 OSC patient samples (Fig. 4A). In addition, ClassNeighbors analysis classified YAP1-expressing
OSC into Class A, which expresses genes associated with DNA recombination, cell cycle and RNA binding (Fig. 5B) and Class B, which expresses genes associated with nucleosome assembly, the respiratory chain, and NADH dehydrogenase activity (Fig. 5C). Class A genes enhance cell cycle-related functions, while Class B genes enhance nucleosome and oxidative phosphorylation pathways. GSEA was performed to investigate significantly enriched pathways that differed between Classes A and B. In Class A, pathways involving tight junctions, WNT and TGF- $\beta$ signaling, and adherens junctions were more active than they were in Class B. In Class B, pathways involving primary immunodeficiency, systematic lupus erythematosus, intestinal immune network for $\operatorname{Ig} \mathrm{A}$ production, regulation of autophagy, and natural killer cellmediated cytotoxicity were enriched (Table III). In Class A, WNT signaling (25) and TGF- $\beta$ signaling (26) were related to cancer progression.

In conclusion, we investigated alterations in YAP1 gene expression in OSC, which was higher than that in 20 other types of cancers. mRNA expression and protein levels of YAP1 were significantly higher in advanced-stage OSC. High YAP and low pYAP were significantly correlated with poor prognosis in OSC. High YAP expression level and also its subcellular distribution may be associated with overall patient survival in OSC.

\section{Acknowledgements}

The present study (research) was supported by the Chungnam National University Hospital Research Fund (2016).

\section{References}

1. Moroishi T, Hansen CG and Guan KL: The emerging roles of YAP and TAZ in cancer. Nat Rev Cancer 15: 73-79, 2015.

2. Mo JS, Park HW and Guan KL: The Hippo signaling pathway in stem cell biology and cancer. EMBO Rep 15: 642-656, 2014.

3. Hong W and Guan KL: The YAP and TAZ transcription co-activators: Key downstream effectors of the mammalian Hippo pathway. Semin Cell Dev Biol 23: 785-793, 2012.

4. Johnson R and Halder G: The two faces of Hippo: Targeting the Hippo pathway for regenerative medicine and cancer treatment. Nat Rev Drug Discov 13: 63-79, 2014.

5. Piccolo S, Dupont S and Cordenonsi M: The biology of YAP/TAZ: Hippo signaling and beyond. Physiol Rev 94: 1287-1312, 2014.

6. Steinhardt AA, Gayyed MF, Klein AP, Dong J, Maitra A, Pan D, Montgomery EA and Anders RA: Expression of Yes-associated protein in common solid tumors. Hum Pathol 39: 1582-1589, 2008.

7. Zender L, Spector MS, Xue W, Flemming P, Cordon-Cardo C, Silke J, Fan ST, Luk JM, Wigler M, Hannon GJ, et al: Identification and validation of oncogenes in liver cancer using an integrative oncogenomic approach. Cell 125: 1253-1267, 2006.

8. Zhao B, Wei X, Li W, Udan RS, Yang Q, Kim J, Xie J, Ikenoue T, $\mathrm{Yu} \mathrm{J}, \mathrm{Li} \mathrm{L}$, et al: Inactivation of YAP oncoprotein by the Hippo pathway is involved in cell contact inhibition and tissue growth control. Genes Dev 21: 2747-2761, 2007.

9. Yu FX and Guan KL: The Hippo pathway: Regulators and regulations. Genes Dev 27: 355-371, 2013.

10. Overholtzer M, Zhang J, Smolen GA, Muir B, Li W, Sgroi DC, Deng CX, Brugge JS and Haber DA: Transforming properties of $Y A P$, a candidate oncogene on the chromosome 11q22 amplicon. Proc Natl Acad Sci USA 103: 12405-12410, 2006.

11. Strano S, Monti O, Pediconi N, Baccarini A, Fontemaggi G, Lapi E, Mantovani F, Damalas A, Citro G, Sacchi A, et al: The transcriptional coactivator Yes-associated protein drives p73 gene-target specificity in response to DNA damage. Mol Cell 18: 447-459, 2005. 
12. Harvey KF, Zhang $X$ and Thomas DM: The Hippo pathway and human cancer. Nat Rev Cancer 13: 246-257, 2013.

13. Xu MZ, Yao TJ, Lee NP, Ng IO, Chan YT, Zender L, Lowe SW, Poon RT and Luk JM: Yes-associated protein is an independent prognostic marker in hepatocellular carcinoma. Cancer 115: 4576-4585, 2009.

14. Wang Y, Dong Q, Zhang Q, Li Z, Wang E and Qiu X: Overexpression of yes-associated protein contributes to progression and poor prognosis of non-small-cell lung cancer. Cancer Sci 101: 1279-1285, 2010.

15. Zhang X, George J, Deb S, Degoutin JL, Takano EA, Fox SB, Bowtell DD and Harvey KF; AOCS Study group: The Hippo pathway transcriptional co-activator, YAP, is an ovarian cancer oncogene. Oncogene 30: 2810-2822, 2011.

16. Xia Y, Chang T, Wang Y, Liu Y, Li W, Li M and Fan HY: YAP promotes ovarian cancer cell tumorigenesis and is indicative of a poor prognosis for ovarian cancer patients. PLoS One 9: e91770, 2014.

17. Jayson GC, Kohn EC, Kitchener HC and Ledermann JA: Ovarian cancer. Lancet 384: 1376-1388, 2014.

18. Kim SI, Lim MC, Lim J, Won YJ, Seo SS, Kang S and Park SY: Incidence of epithelial ovarian cancer according to histologic subtypes in Korea, 1999 to 2012. J Gynecol Oncol 27: e5, 2016.

19. Golub TR, Slonim DK, Tamayo P, Huard C, Gaasenbeek M, Mesirov JP, Coller H, Loh ML, Downing JR, Caligiuri MA, et al: Molecular classification of cancer: Class discovery and class prediction by gene expression monitoring. Science 286: 531-537, 1999.

20. Huang DW, Sherman BT, Tan Q, Collins JR, Alvord WG, Roayaei J, Stephens R, Baseler MW, Lane HC and Lempicki RA The DAVID Gene Functional Classification Tool: A novel biological module-centric algorithm to functionally analyze large gene lists. Genome Biol 8: R183, 2007.
21. Kanehisa M, Goto S, Sato Y, Furumichi M and Tanabe M: KEGG for integration and interpretation of large-scale molecular data sets. Nucleic Acids Res 40: D109-D114, 2012.

22. Kanehisa M and Goto S: KEGG: Kyoto encyclopedia of genes and genomes. Nucleic Acids Res 28: 27-30, 2000.

23. Yang D, Sun Y, Hu L, Zheng H, Ji P, Pecot CV, Zhao Y, Reynolds S, Cheng H, Rupaimoole R, et al: Integrated analyses identify a master microRNA regulatory network for the mesenchymal subtype in serous ovarian cancer. Cancer Cell 23: 186-199, 2013.

24. Bell D, Berchuck A, Birrer M, Chien J, Cramer DW, Dao F, Dhir R, DiSaia P, Gabra H, Glenn P, et al; Cancer Genome Atlas Research Network: Integrated genomic analyses of ovarian carcinoma. Nature 474: 609-615, 2011.

25. DiMeo TA, Anderson K, Phadke P, Fan C, Perou CM, Naber S and Kuperwasser C: A novel lung metastasis signature links Wnt signaling with cancer cell self-renewal and epithelial-mesenchymal transition in basal-like breast cancer. Cancer Res 69: 5364-5373, 2009.

26. Ikushima $\mathrm{H}$ and Miyazono K: TGFbeta signalling: A complex web in cancer progression. Nat Rev Cancer 10: 415-424, 2010.

27. Dong J, Feldmann G, Huang J, Wu S, Zhang N, Comerford SA, Gayyed MF, Anders RA, Maitra A and Pan D: Elucidation of a universal size-control mechanism in Drosophila and mammals. Cell 130: 1120-1133, 2007.

28. Hall CA, Wang R, Miao J, Oliva E, Shen X, Wheeler T, Hilsenbeck SG, Orsulic S and Goode S: Hippo pathway effector Yap is an ovarian cancer oncogene. Cancer Res 70: 8517-8525, 2010. 